\title{
Quantitative analysis of the growth of individual Bacillus coagulans cells by microdroplet technology
}

\author{
Xudong Zhu, Xiang Shi, Ju Chu, Bangce Ye, Peng Zuo* and Yonghong Wang ${ }^{*}$
}

\begin{abstract}
Background: Cellular physiological responses, which are often obscured by inferences from population-level data, are of great importance in cell biology. Microfluidics has emerged as an important tool for biological research on a small scale, reaching even the single-cell level.

Results: In this work, a flow-focusing microdroplet generator was developed to produce monodisperse microdroplets with high stability. Individual B. coagulans cells were encapsulated in the microdroplets and cultured offline. The specific growth rate of $B$. coagulans at the single-cell level was analyzed, and the growth of $B$. coagulans in the droplets showed good consistency with that in flasks, with a correlation coefficient of 0.98 . The morphological heterogeneity and its potential relationship with the production of lactic acid by $B$. coagulans were evaluated using a microscopic imaging method.
\end{abstract}

Conclusion: We have demonstrated a single-cell monitoring methodology based on microdroplets. This approach has great potential for studying a range of behavioral and physiological features of bacteria at the single-cell level.

Keywords: Bacillus coagulans, Microdroplets, Morphological heterogeneity, Single cell

\section{Background}

As a lactic acid-forming bacterial species, B. coagulans has been widely used in the industrial production of lactic acid. Estimated to have reached 367,300 metric tons by 2017, the demand for lactic acid is expected to grow at a yearly rate of almost $20 \%$ due to the increasing demand for polylactic acid (Abdel-Rahman et al. 2013). As a result, significant efforts have focused on maximizing the production of lactic acid by B. coagulans by optimizing growth conditions and developing bioreactors. The field of microbiology has traditionally been concerned with and focused on studies at the population level. Recently, researchers have pursued methods for observing cellular physiological responses, which are often obscured by inferences from population-level data. Microfluidic platforms have emerged as important tools

\footnotetext{
*Correspondence: pzuo@ecust.edu.cn; yhwang@ecust.edu.cn State Key Laboratory of Bioreactor Engineering, College of Biotechnology, East China University of Science and Technology, P.O. Box 329, 130 Meilong Road, Shanghai 200237, China
}

for biological research at a small scale, even down to the single-cell level. Furthermore, due to the low material consumption and monodisperse size of microdroplets, microdroplet technology is a promising tool for $B$. coagulans strain screening (Abalde-Cela et al. 2015; Kim et al. 2015; Lim and Abate 2013). Various microfluidic devices have been introduced for the study of cell cultivation and high-throughput screening (Shen et al. 2014); (Falconnet et al. 2011). Diverse methods of droplet generation and droplet capture have been developed to monitor cell growth (Jakiela et al. 2013; Yu et al. 2014). In this study, a low-cost oil and surfactant combination that was stable under high temperature $\left(50{ }^{\circ} \mathrm{C}\right)$ was developed. For nonspheroid cells, the effective pixel approach provided a simple method for cell counting. In addition, we studied the consistency of cell growth in droplets and in shaking flasks as well as the relationship between phenotype and lactic acid production. Furthermore, the growth of $B$. coagulans from single cells was observed by microscopic imaging. We quantitatively analyzed the growth of 
B. coagulans and found that the growth of B. coagulans within the droplets was consistent with that in flasks. The morphological heterogeneity of $B$. coagulans and its relationship with lactic acid production were studied and it was found that longer B. coagulans cells have a lower productivity of lactic acid.

\section{Methods}

\section{Strains and media}

Bacillus coagulans HL7-A, a derivative of the industrial strain B. coagulans (CGMCC NO. 1.2407) obtained by random mutagenesis, was used in this study. The liquid medium contained $90 \mathrm{~g}$ of glucose $\mathrm{l}^{-1}, 13.33 \mathrm{~g}$ of yeast extract $\mathrm{l}^{-1}, 13.33 \mathrm{~g}$ of tryptone $\mathrm{l}^{-1}, 0.67 \mathrm{~g}$ of $\mathrm{CH}_{3} \mathrm{COONa} \mathrm{l}^{-1}, 0.01 \mathrm{~g}_{\text {of }} \mathrm{NaCl} \mathrm{l}^{-1}, 0.01 \mathrm{~g}$ of $\mathrm{MgSO}_{4} \mathrm{l}^{-1}$, $0.01 \mathrm{~g}$ of $\mathrm{FeSO}_{4} \mathrm{l}^{-1}$, and $0.01 \mathrm{~g}$ of $\mathrm{MnSO}_{4} \mathrm{l}^{-1}$. The $\mathrm{pH}$ was adjusted to 6.0 with $\mathrm{HCl}$ and $\mathrm{NaOH}$, and the medium was sterilized for $20 \mathrm{~min}$ at $115^{\circ} \mathrm{C}$. The glucose was separately sterilized at $115{ }^{\circ} \mathrm{C}$ and mixed with the medium before inoculation.

\section{Culture conditions}

After generation, microdroplets were collected in a 2-ml EP tube and cultured at $50{ }^{\circ} \mathrm{C}$ for $12 \mathrm{~h}$ in an incubator without shaking. In flasks, cells were incubated at $50{ }^{\circ} \mathrm{C}$ with shaking $(200 \mathrm{rpm})$ for $12 \mathrm{~h}$ with a seeding density of approximately $9 \times 10^{7}$ cells ml $^{-1}$. The $\mathrm{pH}$ was not controlled for either culture mode.

\section{Microfluidic system fabrication}

The pattern for the microfluidic device was designed using AutoCAD and was produced on a high-resolution plotted film. This film was bonded to a customized quartz glass, resulting in a compositional photomask. The negative photoresist SU-8 2075 was spin-coated onto a 4-inch silicon wafer. A mask aligner was subsequently used for photoetching and led to a male mould in the silicon wafer. PDMS (polydimethylsiloxane) pre-polymer and curing agent were mixed at a ratio of 10:1 and rapidly stirred. The PDMS mixture was poured onto the silicon master, degassed in a vacuum oven, and then cured at $65{ }^{\circ} \mathrm{C}$ overnight. After curing, the PDMS replica containing the microchannel pattern was peeled away from the silicon master. A hole puncher was used to create holes in a set position to form inlets and outlets for the samples and reagents. The PDMS replica and glass slides were exposed to an oxygen plasma (Harrick Plasma, USA) for $180 \mathrm{~s}$ at $300 \mathrm{~W}$ and were then face-face bonded.

\section{Droplet formation}

For W/O (water in oil) microdroplet formation, paraffin oil (Sigma-Aldrich) containing 3.0\% (w/w) ABIL EM90 surfactant was used as the oil phase. A cell suspension at a concentration of $\sim 3.5 \times 10^{6}$ cells $\mathrm{ml}^{-1}$ was used as the aqueous phase. These two fluids were loaded into $1-\mathrm{ml}$ gas-tight syringes (BD, USA). Two syringe pumps (Chemyx, USA) were used to deliver each phase into the flowfocusing microfluidic device through polyethylene (PE) tubing $(\mathrm{ID}=0.5 \mathrm{~mm})$. The flow rates of $F_{\text {oil }}$ and $F_{\text {aqu }}$ were 200 and $40 \mu \mathrm{l} \mathrm{h}{ }^{-1}$, respectively.

\section{Microscopic imaging of droplets}

The EVOS FL Auto Imaging System (Thermo, USA) was used for microscopic imaging of the droplets. For brightfield microscopic images, droplets were distributed on a glass slide or in the wells of 96-well plates and observed under $400 \times$ magnification. During image acquisition, microscope focusing was first applied to ensure maximum hyphal presentation and then to maintain clarity. Under these criteria, cells of different depths of field can be presented on 2D images. In the worst case, a hypha was exactly perpendicular to the observation plane such that only one point was observed. This situation was easy to avoid because the hyphae show Brownian motion and frequently change direction. Thus, images were not captured until the hyphae reached a maximum imaging length. For pixel statistics, three photos of the same droplet were taken to obtain a mean value.

For fluorescence microscopic images, the $\mathrm{pH}$-sensitive Invitrogen pHrodo Green (Thermo, USA) dye was used to indicate lactic acid production. The optimal absorption and fluorescence emission maxima of the pHrodo Green dye and its conjugates are approximately $509 \mathrm{~nm}$ and $533 \mathrm{~nm}$, respectively. To minimize the impact of background fluorescence of the medium, the DAPI channel was chosen for imaging.

\section{Positioning observation of droplets in 96-well plates}

In this study, wells of 96-well plates were used as vessels for the observation of droplets. Each well was pre-filled with $90 \mu \mathrm{l}$ of the same oil phase used in droplet generation. After droplet generation, a 10- $\mu \mathrm{l}$ emulsion of droplets collected in the EP tube was transferred to the wells by a pipette. Then, the emulsion was gently mixed using the pipette tip. This procedure was performed 3 more times to achieve a gradient dilution of the droplets. Tens of droplets were present in each well, and these droplets were distributed in a single layer, which was conducive to observation. The 96-well plate was gently moved from the incubator to the microscope stage when observation was needed. Due to the high viscosity of the oil phase, the droplets maintained their position during this process.

\section{Statistical analysis method}

The hyphal length in a droplet containing a mass of cells was calculated from the average value of five randomly 


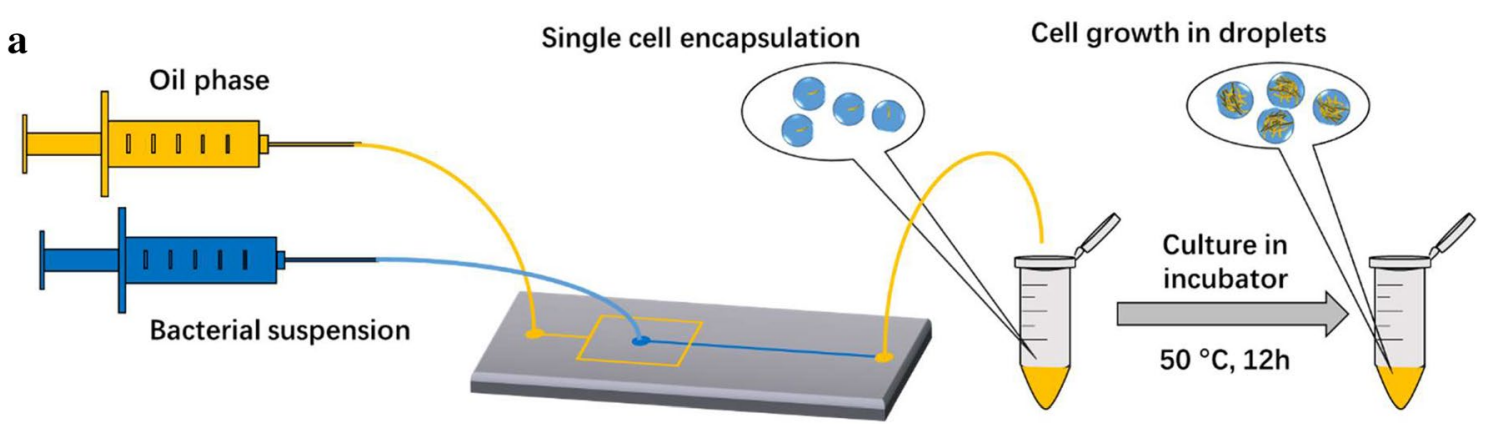

b
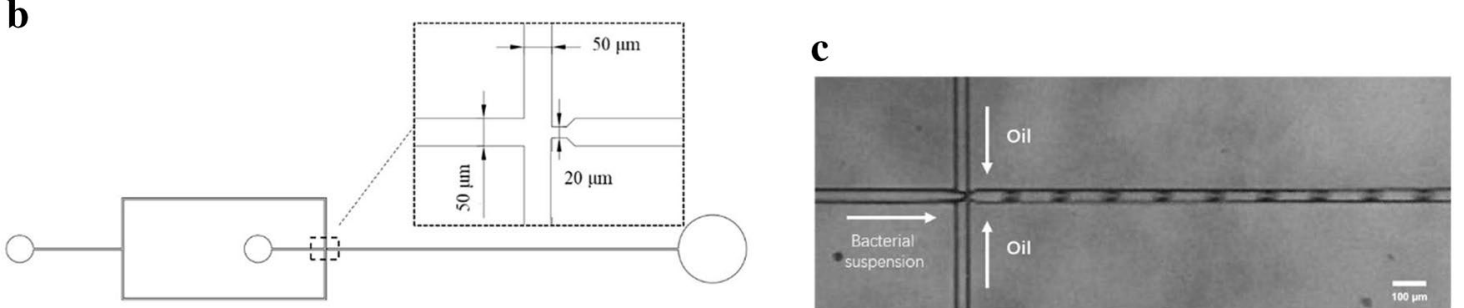

Fig. 1 Encapsulation and growth of B. coagulans in microdroplets. a Schematic illustration of droplet generation and off-chip cultivation. The device had a uniform channel depth of $25 \mu \mathrm{m}$. Microdroplets were generated in a flow-focusing microfluidic device and collected in an EP tube for incubation at $50^{\circ} \mathrm{C}$ for $12 \mathrm{~h}$. $\mathbf{b}$ The blueprint and dimensions of the flow-focusing microfluidic chip. All channels had a uniform depth of $25 \mu \mathrm{m}$. c A bright-field microscopy image showing the generation of microdroplets. The bacterial suspension flow and oil flow are indicated by white arrows. Scale bar: $100 \mu \mathrm{m}$

selected hyphae in a given droplet. The standard deviations were also calculated and are shown as error bars. According to the length of the hyphae, the droplets were divided into three groups: $<5 \mu \mathrm{m}, 5-10 \mu \mathrm{m}$ and $>10 \mu \mathrm{m}$. For fluorescence measurements, all droplets were photographed using the same microscope parameters. Then, the fluorescence images were converted into gray-scale images, and the gray values were counted. The average gray values of all the droplets in each group were calculated and compared. The standard deviations were also calculated and are shown as error bars.

\section{Results and discussion W/O droplet generation}

In this section, the microdroplet was used as an individual vessel to investigate the growth of $B$. coagulans at the single-cell level. A flow-focusing microfluidic device was used to generate microdroplets with a low polydispersity (Fig. 1). As the incubation temperature of B. coagulans reached $50^{\circ} \mathrm{C}$, obtaining droplets with long-term stability was challenging. By extensively screening diverse oils and surfactants, we found that paraffin oil (Sigma, USA) containing 3.0\% (w/w) ABIL EM90 surfactant could produce droplets with long-term stability.

For cell culture in flasks, the seeding density of B. coagulans was $\sim 9 \times 10^{7}$ cells $\mathrm{ml}^{-1}$. To maintain a constant seeding density, the diameter of a droplet containing one cell should be $27.4 \mu \mathrm{m}$ based on theoretical calculations. The size of the droplets could be controlled by the diameter of the generating nozzle, as well as the flow rate ratio of the different phases. Here, using flow rates of $200 \mu \mathrm{h} \mathrm{h}^{-1}\left(F_{\text {oil }}\right)$ and $40 \mu \mathrm{h}^{-1}\left(F_{\text {aqu }}\right)$ and a generating nozzle with dimensions of $20 \mu \mathrm{m} \times 25 \mu \mathrm{m}$ (width $\times$ depth), monodisperse droplets of $\sim 28 \mu \mathrm{m}$ in diameter $(\sim 12 \mathrm{pl}$ in volume) (Fig. 2a) were formed. The microdroplets were stable and did not fuse with each other whilst being transferred into the EP tube and cultured at $50{ }^{\circ} \mathrm{C}$ for $12 \mathrm{~h}$ (Fig. 2b).

The number of cells in each droplet follows a Poisson distribution (Hosokawa et al. 2015), which was dependent on the incoming cell density. Herein, a bacterial suspension at the exponential growth phase ( $6 \mathrm{~h}$ of growth in a flask) was diluted with fresh medium to a concentration of $\sim 3.5 \times 10^{6} \mathrm{cells} \mathrm{ml}^{-1}$. The suspension was then introduced into the microdroplet device to generate a suspension of droplets in oil. Under these conditions, approximately $22 \%$ of the microdroplets contained only one cell (Fig. 2c, d).

\section{Quantitative analysis of the growth of B. coagulans in the microdroplets}

The growth of B. coagulans cells encapsulated in microdroplets was monitored by microscopic imaging at 
a
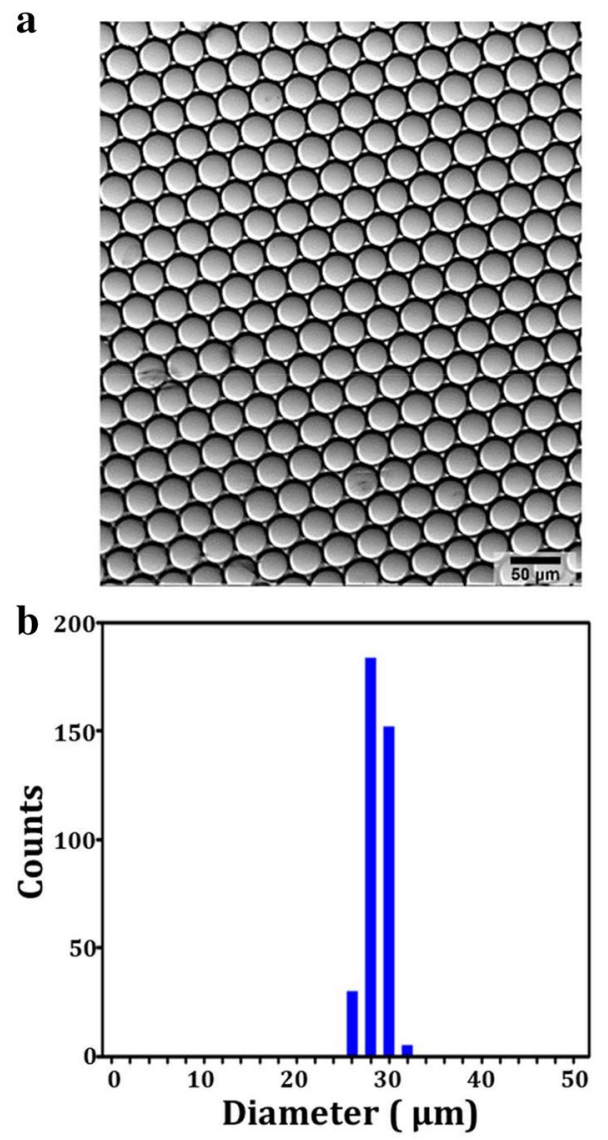

c

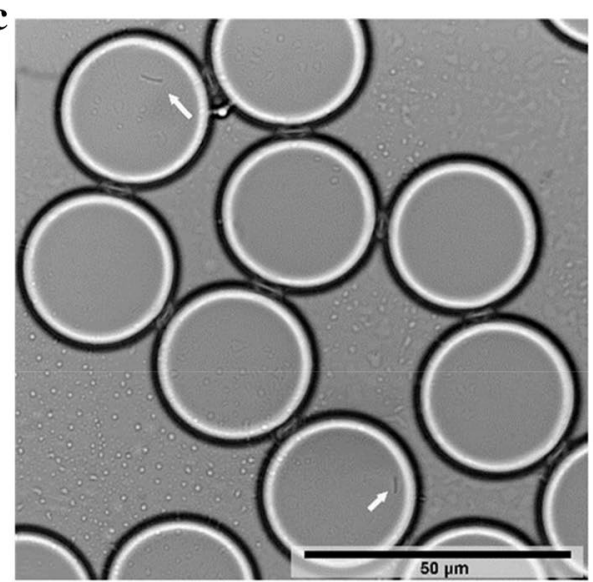

d 500

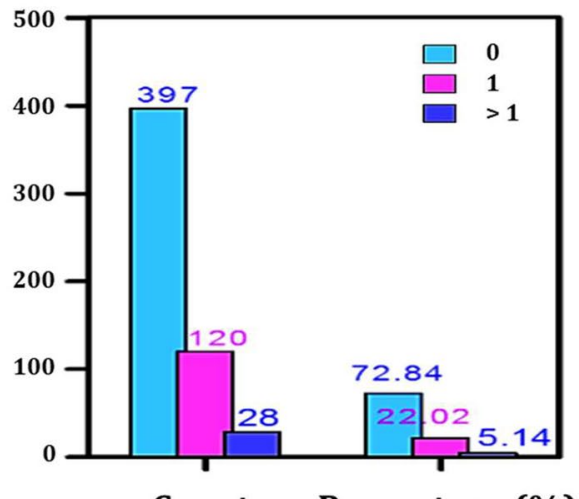

Fig. 2 Generation of W/O (water in oil) microdroplets by a microfluidic droplet generator. a Microphotograph of microdroplets generated by a microfluidic droplet generator. Scale bar: $50 \mu \mathrm{m}$. b Distribution of the microdroplet diameters. The overall variation coefficient is $3.69 \%$. c A bright-field microscopy image showing B. coagulans (highlighted by white arrows) being encapsulated in microdroplets. Scale bar: $50 \mu \mathrm{m}$. $\mathbf{d}$ Initial number of cells per droplet. More than 500 droplets were investigated immediately after generation. The number and percentage of droplets containing 0, 1 and more than 1 cells are listed

different incubation times. Five droplet images were randomly selected at different time points and are presented together artificially for better comparison, as shown in Fig. 3 . These images clearly show the growth of $B$. coagulans in the microdroplets over $12 \mathrm{~h}$ after encapsulation. During the first $2 \mathrm{~h}$, the bacteria grew in length. After $4 \mathrm{~h}$ of incubation, the parent bacteria were divided into several daughter bacteria. At $6-10 \mathrm{~h}$, the bacteria were in the exponential growth phase. After $12 \mathrm{~h}$ of incubation, the amount of bacteria changed little compared to $10 \mathrm{~h}$, indicating that the bacteria had reached the stationary phase.

A positioning observation was performed to obtain a quantitative analysis of the growth of $B$. coagulans from single cells. Here, droplets were collected in wells of a 96-well plate at a concentration of tens of droplets per well by gentle gradient dilution. Figure $4 \mathrm{a}$ presents images of the cells in the microdroplets under a brightfield microscope, acquired every $2 \mathrm{~h}$.
Due to the length of B. coagulans, it was difficult to count the number of bacteria. Instead, the number of effective pixels (pixels occupied by hyphae in the microscope image) was counted to realize a quantitative tracking of the growth of individual $B$. coagulans in a microdroplet (Additional file 1: Fig. S1). Figure 4b shows the effective pixels (a mean value of 5 droplets) at different time points. It was found that the cells in the droplets experienced an adaptation period (2-4 h), an exponential growth period $(4-10 \mathrm{~h})$ and a stationary phase $(10-12 \mathrm{~h}$ ), as observed in the flask (Additional file 1: Fig. S2). The specific growth rate $(\mu)$ of the cells within the droplets was calculated and compared with that in shaking flasks with the same seeding density. The results are shown in Table 1 . We found that the specific growth rate of the bacteria in the droplets was similar to that in the flasks.

The correlation coefficient for the number of cells in the droplet (characterized by the number of effective pixels) 


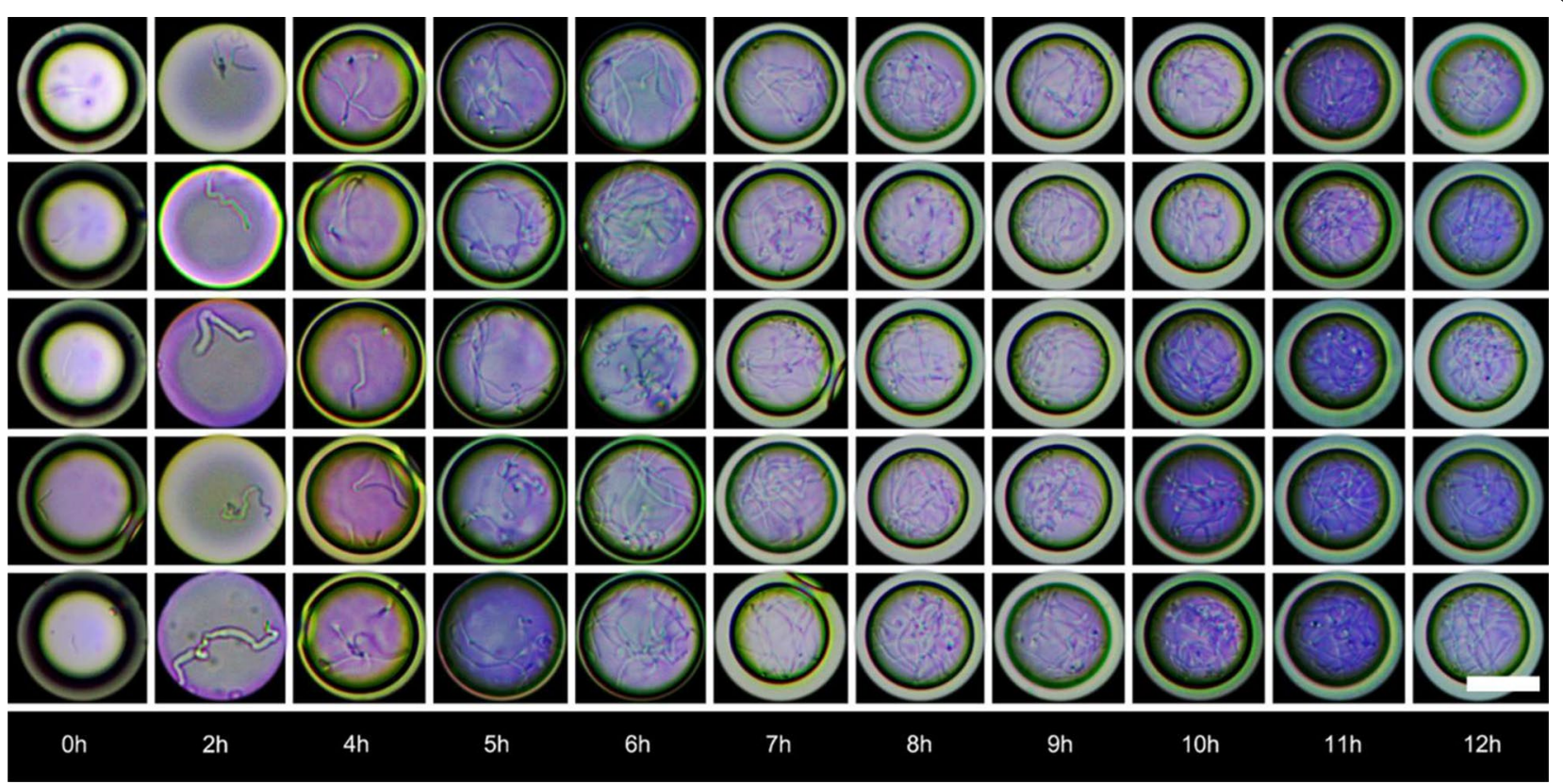

Fig. 3 The growth of $B$. coagulans cells in microdroplets at $50{ }^{\circ} \mathrm{C}$ for $12 \mathrm{~h}$. Bright-field images show the increase in length and the division of $B$. coagulans. Scale bar: $20 \mu \mathrm{m}$

and that in the flasks was determined and was found to reach 0.98 (Fig. 4c). This result indicates that microdroplets can be used as small bioreactors for B. coagulans instead of shaking flasks. Combining the features of small volume (picoliter) and low variability in size (less than $3 \%)$, the microdroplets show potential for high-throughput cell culturing and screening for B. coagulans, with valuable characteristics such as high lactic acid yield.

\section{Morphological heterogeneity of B. coagulans}

In this study, a single colony of $B$. coagulans that was subcultured for twenty generations in agar slants showed obvious morphological heterogeneity. This type of single colony was incubated in a flask for seed culture and was then encapsulated in the droplets. The cells were consistent in size within the droplets when initially encapsulated. However, microscopic images after $6 \mathrm{~h}$ of incubation at $50{ }^{\circ} \mathrm{C}$ showed a significant difference in the mycelium length of B. coagulans, as shown in Fig. 5a.

The $\mathrm{pH}$ fluorescence indicator pHrodo Green (Thermo, USA) was added to the medium before droplet generation to determine whether there is a relationship between the morphological character and the production of lactic acid. The fluorescence intensity of pHrodo increases with decreasing $\mathrm{pH}$. Thus, a higher fluorescence intensity in the droplets represents a higher lactic acid concentration (Additional file 1: Fig. S3). Figure 5b presents a merged image of bright field and fluorescence microscopic images obtained after $6 \mathrm{~h}$ of incubation at $50{ }^{\circ} \mathrm{C}$, where the fluorescence is shown in blue. It is obvious that the shorter B. coagulans cells produced more lactic acid than the longer cells.

Statistics on hyphal length and fluorescence intensity for one hundred droplets were acquired. In Fig. 5c, the length of the hyphae in one droplet is shown in columns. This value represents the average of five randomly selected hyphae in a given droplet. According to the length of the hyphae, the droplets were divided into three groups: $<5 \mu \mathrm{m}, 5-10 \mu \mathrm{m}$ and $>10 \mu \mathrm{m}$. The fluorescence intensity of the droplets was converted into a gray value for analysis. The square symbols in Fig. 5c present the average gray values of all the droplets in each group. The results indicate that the gray value is negatively correlated with hyphal length. Hence, the shorter B. coagulans cells produced more lactic acid than the longer cells.

\section{Conclusion}

Due to the advantage of microdroplets in single-cell compartmentalization, the presented experiment visually demonstrated the growth of $B$. coagulans at the single-cell level. The growth of $B$. coagulans was quantified by microscopic image analysis. Statistical results showed that the growth status of the bacteria in the microdroplets was consistent with that in flasks, with the correlation coefficient reaching 0.98 . This result demonstrates the feasibility of using microdroplets for high-throughput screening for B. coagulans (Maerkl 2009). Moreover, the morphological heterogeneity 

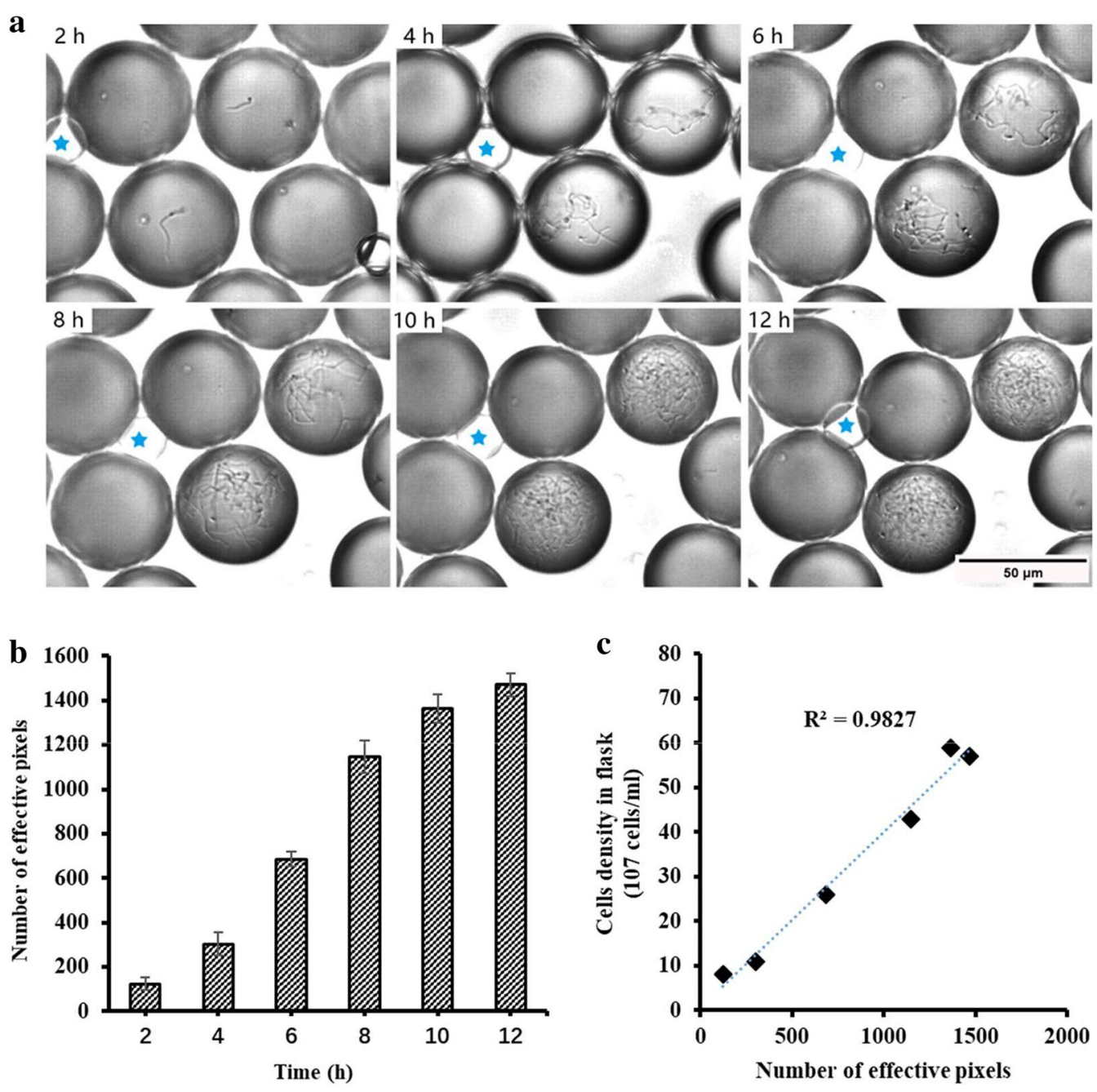

Fig. 4 Quantitative analysis of the growth of individual B. coagulans cells. a Fixed observation of individual B. coagulans cells. The microdroplets shown in each image are the same, and the smaller microdroplet marked by a blue star was used as a reference. Scale bar: $50 \mu \mathrm{m}$. $\mathbf{b}$ The growth of B. coagulans was quantified by counting the number of effective pixels. $\mathbf{c}$ Scatterplot of the cell density in the flask and the effective pixels in the microdroplets. The results show a good consistency, with an $R_{2}$ value of 0.98

Table 1 Specific growth rate of $B$. coagulans in droplets and flasks

\begin{tabular}{lllllll}
\hline Time $(\mathbf{h})$ & $\mathbf{2}$ & $\mathbf{4}$ & $\mathbf{6}$ & $\mathbf{8}$ & $\mathbf{1 0}$ & $\mathbf{1 2}$ \\
\hline$\mu(\mathrm{h})$ in droplets & $0.02(0.018)^{*}$ & $0.45(0.114)$ & $0.41(0.122)$ & $0.34(0.043)$ & $0.09(0.085)$ & $0.04(0.037)$ \\
$\mu(\mathrm{h})$ in flasks & $0.03(0.011)$ & $0.48(0.083)$ & $0.47(0.078)$ & $0.36(0.091)$ & $0.10(0.063)$ & $0.09(0.015)$ \\
\hline
\end{tabular}

* Standard deviations are shown in parentheses

of $B$. coagulans derived from a single colony was presented. It was found that shorter B. coagulans cells produced more lactic acid than longer cells. In addition to the biotechnological applications described here, this methodology can be expanded to the culture of other cell lines by altering the environmental parameters. The 


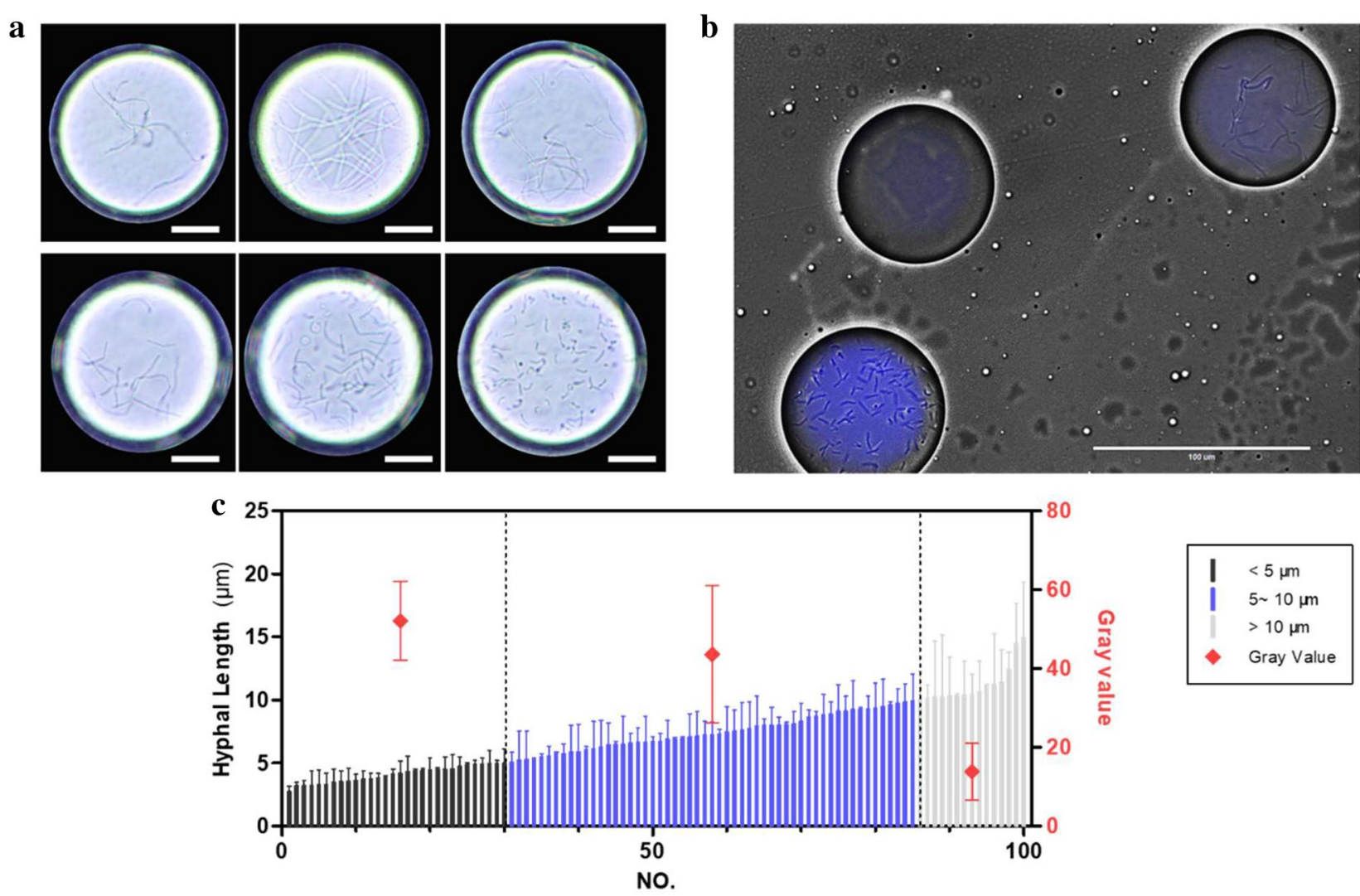

Fig. 5 Morphological heterogeneity of B. coagulans derived from a single colony. a There was a significant difference in hypha length. Scale bar: $20 \mu \mathrm{m}$. b The shorter B. coagulans cells (lower left corner) produced more lactic acid than the longer cells (top right corner). Scale bar: $100 \mu \mathrm{m}$. c Relationship between the hyphal length and lactic acid productivity of $B$. coagulans. The length of the hyphae in one droplet is shown in the columns, representing the average length of five randomly selected hyphae in a given droplet. The square symbol for each group is the average gray value for all the droplets in the group. The results show that the shorter B. coagulans cells produced more lactic acid than the longer cells

system also represents a method for studying a range of behavioral and physiological features of bacteria at the single-cell level.

\section{Additional file}

Additional file 1: Fig. S1. The method of pixels counting. (A) Original photo of the droplets. (B) Adjusted image by adjusting the "threshold" and the pixels were automatically counted. Fig. S2. The growth curve of Bacillus coagulans in shake flask without (black) and with pHrodo (red). The data were obtained from three parallel experiments for each. Fig. $\mathbf{S 3 .}$ Lactic acid assay in microdroplets. (A) Fluorescence imaging of microdroplets containing broth with a range of lactic acid, $8 \mathrm{~g} / \mathrm{L}$ (I), $11 \mathrm{~g} / \mathrm{L}$ (II), $12 \mathrm{~g} / \mathrm{L}$ (III), $14 \mathrm{~g} / \mathrm{L}$ (IV). (B) The corresponding average gray value derive from the fluorescence intensity of the microdroplets in the images of Fig. S1 A.

\section{Abbreviations}

ID: inner diameter; PDMS: polydimethylsiloxane; PLA: polylactic acid; W/O: water in oil.

\section{Authors' contributions}

$X Z$ and $X S$ designed the experiments. XZ and XS performed the experiments. $X Z$ drafted the manuscript. PZ, YW, JC and BY assisted in the research experiments. All authors read and approved the final manuscript.

\section{Acknowledgements}

Not applicable.

\section{Competing interests}

The authors declare that they have no competing interests.

\section{Availability of data and materials}

All data generated or analyzed during this study are included in this article.

\section{Consent for publication}

All authors have read this article and have approved its submission to Bioresources and Bioprocessing.

\section{Ethics approval and consent to participate}

Not applicable. 


\section{Funding}

This work was supported by the National Science Foundation for Young Scientists of China (31700038) and the National Key Research and Development Program (2017YFB0309302).

\section{Publisher's Note}

Springer Nature remains neutral with regard to jurisdictional claims in published maps and institutional affiliations.

Received: 20 July 2018 Accepted: 3 October 2018

Published online: 16 October 2018

\section{References}

Abalde-Cela S, Gould A, Liu X, Kazamia E, Smith AG, Abell C (2015) Highthroughput detection of ethanol-producing cyanobacteria in a microdroplet platform. J R Soc Interface 12(106):0216

Abdel-Rahman MA, Tashiro Y, Sonomoto K (2013) Recent advances in lactic acid production by microbial fermentation processes. Biotechnol Adv 31(6):877-902

Falconnet D, Niemistoe A, Taylor RJ, Ricicova M, Galitski T, Shmulevich I, Hansen CL (2011) High-throughput tracking of single yeast cells in a microfluidic imaging matrix. Lab Chip 11(3):466-473
Hosokawa M, Hoshino Y, Nishikawa Y, Hirose T, Yoon DH, Mori T, Sekiguchi T, Shoji S, Takeyama H (2015) Droplet-based microfluidics for highthroughput screening of a metagenomic library for isolation of microbial enzymes. Biosens Bioelectron 67:379-385

Jakiela S, Kaminski TS, Cybulski O, Weibel DB, Garstecki P (2013) Bacterial growth and adaptation in microdroplet chemostats. Angew Chem Int Ed 52(34):8908-8911

Kim HS, Devarenne TP, Han A (2015) A high-throughput microfluidic singlecell screening platform capable of selective cell extraction. Lab Chip 15(11):2467-2475

Lim SW, Abate AR (2013) Ultrahigh-throughput sorting of microfluidic drops with flow cytometry. Lab Chip 13(23):4563-4572

MaerkI SJ (2009) Integration column: microfluidic high-throughput screening. Integr Biol 1(1):19-29

Shen HH, Tsai HY, Yao DJ (2014) Single mouse oocyte encapsulated in medium-in-oil microdroplets by using a polydimethylsiloxane microfluidic device. Sensors Mater 26(2):85-94

Yu JQ, Chin LK, Lei L, Lin ZP, Ser W, Chen H, Ayi TC, Yap PH, Chen CH, Liu AQ (2014) Droplet optofluidic imaging for lambda-bacteriophage detection via co-culture with host cell Escherichia coli. Lab Chip 14(18):3519-3524

\section{Submit your manuscript to a SpringerOpen ${ }^{\odot}$ journal and benefit from:}

- Convenient online submission

- Rigorous peer review

- Open access: articles freely available online

- High visibility within the field

- Retaining the copyright to your article

Submit your next manuscript at $\boldsymbol{\nabla}$ springeropen.com 